\title{
Fronteras paradójicas: contrastes de desarrollo en la frontera México-EUA
}

\author{
Raúl Fernández \\ Universidad de California en Irvine
}

\begin{abstract}
Resumen
La unidad llamada "la frontera" en realidad está caracterizada por grandes diferencias y heterogeneidades. Dentro de esta multiplicidad de facetas se pueden observar muchos otros ejemplos de paradojas del desarrollo; conflictos y contrastes que pueden servir para aprender y para difundir el conocimiento sobre esta parte del continente. La frontera es como una lucha de tensiones; el conocimiento de las diferentes facetas de estas tensiones conducirá a un mejoramiento en el grado de precisión en lo referente a las tendencias actuales en la región. De acuerdo a lo expuesto en este trabajo se puede afirmar que aunque el gobierno de México ha tratado de controlar el área fronteriza económica y políticamente, el probable incremento del poder económico norteamericano en la región y el mayor peso económico de la zona fronteriza norte, podrían conducir a una inversión de papeles en un futuro. Es decir, los desarrollos en la frontera serán por algún tiempo la clave para comprender los desarrollos a nivel nacional; y no a la inversa.
\end{abstract}

Palabras clave: sociedades contrastantes, desarrollo nacional, frontera México-Estados Unidos, ciudades fronterizas gemelas.

\begin{abstract}
The unity called "the border" is rather characterized by differences and heterogeneities. Many examples of paradoxes, conflicts, and contrasts can be observed within this multiple facets, which could be useful to spread the knowledge about this part of the continent. The border is like a struggle of tensions, and understanding the diverse aspects of these tensions will lead to a more accurate degree of precision regarding current trends on this region. As this work describes herein, the potential increase of American economic power over the region, and the stronger economical influence of the North border zone could trend to an exchange of roles in the future; regardless the Mexican government has tried to establish political and economical control over the border zone. It means that border developments will eventually be the key to understand national development, and not the opposite.
\end{abstract}

Keywords: contrasting societies, national development, Mexico-United States border, twin border cities. 


\title{
FRONTERAS PARADOJICAS: CONTRASTES DE DESARROLLO EN LA FRONTERA MEXICO-EUA *
}

\author{
Por \\ Raúl Fernández**
}

Cuando se escribe o se habla sobre la frontera México-Estados Unidos - por ejemplo en frases tales como "economía de frontera", "la región fronteriza", etcétera-, los autores, incluso yo, frecuentemente damos la impresión de que se hace referencia a una unidad social, geográfica o económica particular. Sin embargo, cuando se empieza a preguntar sobre los elementos que llegan a formar tal unidad, la falta de homogeneidad se hace evidente. Existe por supuesto, la correspondencia política regional en el sentido de que, a lo largo de los kilómetros de línea divisoria internacional, dos naciones se encuentran cara a cara: una, la mayor potencia industrial del mundo; la otra, una de las más grandes deudoras. Más allá de esto, la frontera se presenta fragmentada en muchas partes contrastantes, cada una de ellas con características individuales y únicas.

La frontera está compuesta de varios centros urbanos importantes, así como de grandes áreas rurales e incluso de zonas desérticas escasamente pobladas. Al finalizar la línea internacional al este, se encuentra el puerto de Brownsville, que presta servicios no sólo al Valle del Río Grande en Texas, sino también a Matamoros, Reynosa, Nuevo Laredo y Monterrey, en México. En el límite oeste, Tijuana, la ciudad "postindustrial", ha hecho del turismo una de sus fuentes de ingresos más importantes. ${ }^{1}$ No muy lejos, la ciudad de Mexicali descansa en el centro de uno de los valles agrícolas más desarrollados y mecanizados del mundo, lo cual da a este centro urbano sus características particulares.

Las ciudades fronterizas de México están conectadas con la capital del país por carreteras y ferrocarril; pero los lazos entre ellas son mucho más débiles. Un habitante de Mexicali que desea viajar a la ciudad de EI Paso por territorio mexicano, puede encontrar dificultades para hacerlo por tren o autobús; por lo tanto, debe tomar la alternativa de cruzar a los Estados Unidos para llegar a El Paso por tierra. Regionalmente hablando, la frontera por el lado mexicano se encuentra fragmentada en tres secciones, todas separadas pero conectadas con los centros nacionales; estas secciones están unidas ya que tienen vínculos económicos con la nación del norte, que presenta una integración más fuerte en su zona fronteriza.

- Traducido por Guadalupe Ortega Villa.

* Program in Comparative Culture, University of California, Irvine, EUA

Documento presentado en el Consejo de la Costa del Pacífico sobre Estudios Latinoamericanos, Las Vegas, Nevada. 19 de octubre de 1985.

1 John A. Price. Tijuana: Urbanization in a Border Culture. University of Notre Dame Press. 1973. 
La unidad que llamamos la frontera, en realidad está caracterizada por grandes diferencias y heterogeneidades. Dentro de esta multiplicidad de facetas podemos observar muchos otros ejemplos de paradojas del desarrollo; conflictos y contrastes que pueden servir al investigador para aprender, $y$ al profesor para difundir el conocimiento sobre esta parte del continente. En muchos sentidos, la frontera es como una lucha de tensiones cambiantes; el conocimiento de las diferentes facetas de estas tensiones conducirá a un mejoramiento en el grado de precisión en lo referente a las tendencias actuales en la región. ${ }^{2}$

\section{PASADO Y PRESENTE}

Los estudiosos de la frontera están plenamente conscientes de los grandes cambios que han ocurrido en los últimos 40 años. Los años de 1945-1985 presenciaron las profundas transformaciones de la base económica, así como las del paisaje geográfico de la faja del territorio mexicano que se extiende de Tijuana-San Diego, a Brownsville-Matamoros. La naturaleza de estas modificaciones constituye una clave para la comprensión de la situación actual. Comprender cómo surgieron los contrastes entre 1945 y 1985 proporciona las bases para especular con fundamento acerca de las tendencias futuras.

En 1945, la zona fronteriza de México no era precisamente un centro de atención para el gobierno ni para los científicos sociales. Sus ciudades y poblados eran pequeños; la industria, el comercio y el turismo tenían poca significancia; la migración a través de la línea internacional era limitada y regulada. Los discursos oficiales de ambos lados de la frontera eran de poco significado político o estratégico.

Para 1985 se "volteó la tortilla". Las ciudades fronterizas se convirtieron en grandes centros urbanizados de rápido crecimiento, se localizaron allí un gran número de firmas industriales, la mayoría procedentes de los Estados Unidos. El Programa Industrial Fronterizo (PIF) iniciado en pequeña escala a finales de los sesenta, proporcionó mucho del empleo industrial en la región, y produjo miles de millones de dólares anuales en artículos de consumo manufacturados. El turismo se transformó en el soporte de varias ciudades fronterizas; el comercio, incluyendo los negocios al menudeo del lado norteamericano, se benefició e incrementó rápidamente con el crecimiento demográfico al sur de la frontera; la migración pasó a ser verdaderamente masiva.

El patrón de desarrollo regional en los Estados Unidos fue un factor importante que influyó en los cambios que condujeron a estos contrastes cuatro décadas después. El crecimiento del sur de California en particular, está íntimamente relacionado con las modificaciones demográficas e

\footnotetext{
2 Cf., Niles Hansen. The Border Economy. Austin, University of Texas Press. 1981.
} 
industriales en la parte oeste de la frontera México-EUA. Los Angeles, por ejemplo, creció de aproximadamente un millón de habitantes al finalizar la Segunda Guerra Mundial, a la actual área metropolitana de 10 millones de habitantes, convirtiéndose en un centro industrial de primer orden con las industrias aeronáuticas, química, de acero y plástico, como sus sectores manufactureros lideres.

Los estados del oeste como California, Nevada, Oregon y Washington, durante este período se apartaron de la norma nacional en cuanto al crecimiento del empleo agrícola. De 1938 a 1959 el empleo agrícola en los Estados Unidos fue drásticamente reducido a la mitad; sin embargo, en estos cuatro estados se duplicó. Fuertes inversiones de capital, especialmente en irrigación, abrieron grandes áreas al cultivo. Este desarrollo industrial y agrícola estimuló el flujo migratorio constante a California y todo el oeste, proveniente del este de EUA y de México. El crecimiento económico de los valles Central, San Joaquín e Imperial, sirvió como imán que atrajo a millones de trabajadores agrícolas mexicanos que con el tiempo se establecieron en los Estados Unidos o en las ciudades fronterizas de México. La industria de Los Angeles, finalmente pudo separar y enviar algunas de sus ramas manufactureras con mayor uso intensivo de mano de obra hacia los centros urbanos al sur de la frontera, especialmente Tijuana, Tecate y Mexicali, lo cual constituyó un cambio en la actividad industrial, y que descansaba en la idea del Programa Industrial Fronterizo, de crear plantas maquiladoras en la frontera.

Por otro lado, en el extremo este de la línea fronteriza, en Texas, se ponía en marcha un patrón alternativo de desarrollo económico regional. También Texas creció rápidamente después de la Segunda Guerra Mundial, pero aquí el foco de crecimiento se localizó en los campos petroleros en la parte norte del estado, lejos de la frontera con México, en una industria que generalmente es intensiva en capital. La industria petroquímica, también de capital intensivo, se desarrolló en el área de la costa del golfo de México conectada con los puertos de Galveston y Corpus Christi. Los cambios en la agricultura también siguieron un patrón distinto que el del oeste, con énfasis en cosechas de agricultura extensiva, como el algodón y la soya. No es sorprendente que el desarrollo de las ciudades mexicanas fronterizas con Texas nunca hayan alcanzado la magnitud, en términos de tamaño y crecimiento, que caracteriza a los centros urbanos mexicanos adyacentes a California; el turismo que por un momento convirtió a Tijuana en una especie de monocultura urbana, no aparece de forma tan pronunciada en las ciudades gemelas de Texas-México. ${ }^{3}$

Si el desarrollo regional de los Estados Unidos juega un papel importante en la definición de los patrones económicos y de cambio social en la frontera mexicana, ¿qué augura el futuro a este respecto?. El enorme

s Véase: John W. House. Frontier on the Rio Grande. Oxford: Clarendon Press. 1982. 
crecimiento industrial del sur de California en los cuarenta, cincuenta y sesenta, ya ha llegado a su fin. A fines de la década de 1970 la industria manufacturera empezó a declinar en el sur de California, y para 1981-1982 el estado se había unido a la lista de los principales estados de la Unión Americana que cerraban sus plantas; fenómeno antes sólo asignado al denominado "cinturón congelado". En los últimos tres años, varios estudios pronosticaron que la industria, la agricultura y las oportunidades de empleo que estos sectores proporcionan, experimentarían un crecimiento limitado en Los Angeles y el sur de California. Generalmente, se espera que para los próximos años el crecimiento en la región denominada "cinturón soleado" mantendrá los mismos niveles, si es que no decrece.

Sobre esta base, es razonable afirmar como una primera aproximación, que la tasa de crecimiento de la parte oeste de la frontera mexicana puede presentar un desaceleramiento similar en su crecimiento económico, demográfico e industrial en el futuro inmediato. Desde luego, se debe tener cuidado al establecer este tipo de correlaciones; me permito agregar que pueden entrar en juego factores que influyen en dirección opuesta. Así, mientras los pronósticos sobre creación de empleo en el oeste y en el "cinturón soleado" podrían ser los menos optimistas de todos los tiempos; los sectores que dependen de la mano de obra barata, tales como el programa de plantas maquiladoras, quizá continuarán estableciendo sus fábricas en las ciudades fronterizas.

\section{ECONOMIA Y POLITICA}

El equilibrio económico de los estados del suroeste transformó en una cuestión política de primer orden el problema de la migración indocumentada de mano de obra mexicana y de otros países hacia los Estados Unidos. En años anteriores, cuando la demanda de fuerza de trabajo había sido mayor, los migrantes indocumentados habían sido silenciosamente bienvenidos (políticamente hablando) en los Estados Unidos. A medida que empezó a decrecer la demanda de fuerza de trabajo en la industria y en la agricultura, creció la intensidad del conflicto entre la gran industria y el capital agrícola por mantener una oferta permanente de mano de obra indocumentada barata por un lado; y por el otro, los dirigentes políticos locales y nacionales que oficialmente representan la fuerza de trabajo de los americanos nativos.

La tensión entre los aspectos económico y político de la migración mexicana a Estados Unidos, representa otro ingrediente de peso en el proceso social de la frontera. Esta se encuentra en el núcleo del atolladero legislativo de 1984, y en el actual debate del Congreso, acerca de una gran reforma a las leyes de inmigración de los Estados Unidos. Los patrones de desarrollo regional que hemos descrito, aunados al severo decrecimiento de la economía estadounidense a principios de la década de los ochenta, contribuyeron a hacer más "visibles" a los trabajadores indocumentados. 
El problema de la disponibilidad de abundantes suministros de mano de obra, en el suroeste de los Estados Unidos, ha preocupado, durante décadas, a los grandes propietarios agrícolas. La economía de los grandes negocios agrícolas determina que la industria tenga garantizada una abundante reserva de fuerza de trabajo, algunas veces a corto plazo. Hubo un tiempo en que los intereses de los negocios agrícolas utilizaron mano de obra extranjera barata como una llave de agua que podían abrir y cerrar; por ejemplo, los períodos de importación de fuerza de trabajo china y japonesa a fines del siglo XIX y principios del XX, así como el Programa de Braceros de los años cincuenta y principios de los sesenta. Pero los acontecimientos de los últimos 40 años, como son la migración masiva de mexicanos, el crecimiento demográfico de las ciudades fronterizas mexicanas, el incremento de la población chicana con lazos de parentesco en México; dieron por resultado que las medidas de control fronterizo y de migración resultaron obsoletas. Asimismo, los recientes cambios en dirección y en ritmo de las tendencias regionales provocaron quejas por parte de los trabajadores nativos contra los trabajadores indocumentados "que quitan los empleos". Si se es un trabajador nativo en Los Angeles o en Tucson, ya sea chicano, negro o anglo, es fácil creer, sea o no realmente cierto, que la causa del desempleo y la inseguridad en el trabajo en un sentido inmediato, se debe a la competencia deshonesta del compañero trabajador de otro país, aunque indocumentado.

La preocupación creciente sobre la inestabilidad en América Central agregó una dimensión multilateral al aspecto político del problema. Ciertamente, debido al intento de la Unión Soviética y sus aliados cubanos de utilizar la inconformidad social de la región con el fin de promover sus propios intereses expansionistas, el conflicto entre las dos superpotencias - EUA y la URSS - pasó a formar parte del debate de la reforma de inmigración.

La reforma de inmigración que el Congreso de los Estados Unidos ha discutido por tres años hasta hoy, tiende a obtener un "equilibrio" entre las demandas económicas - políticamente poderosas- de los grandes negocios del capital agrícola; los esfuerzos de los sindicatos que no están dispuestos a permitir la importación controlada de mano de obra barata, y la percepción de un reclamo por parte de los trabajadores nativos en conra de la competencia desleal que representan los trabajadores indocumentados. Este "equilibrio" apunta, sin lugar a dudas, hacia un control sociopolítico indirecto de los trabajadores indocumentados actualmente en los Estados Unidos, al establecimiento de restricciones sobre mano de obra indocumentada adicional y a la repatriación indirecta pero objetiva de algunos de los trabajadores actualmente indocumentados, al tiempo que se garantiza una oferta permanente de fuerza de trabajo muy al estilo del viejo Programa Bracero, a 
fin de satisfacer las necesidades de fuerza de trabajo agrícola en el suroeste. $^{4}$

Resulta probable que la legislación restrictiva sobre migración, sea aprobada por el Congreso de los Estados Unidos. La última vez que el gobierno de los Estados Unidos pudo suspender eficazmente la migración proveniente de México en cantidades sustanciales - cuando se efectuó la suspensión del Programa Bracero en 1964-, se hicieron sentir repercusiones importantes en la frontera mexicana. Tres años después de la conclusión del programa, se reportaba que trabajadores del interior de México aún arribaban a la frontera con la esperanza de ser contratados como braceros. El desempleo en las ciudades de la frontera aumentó explosivamente. El gobierno mexicano, incapaz de manejar el problema mediante sus propios recursos nacionales, instrumentó un acuerdo especial con el fin de aliviar (muy posiblemente) el problema del desempleo en su frontera. Las restricciones en torno a la inversión extranjera fueron hechas a un lado, y con la cooperación del Departamento de Comercio de los Estados Unidos, a industriales de ese país les fue permitido establecer plantas maquiladoras al sur de la frontera - el Programa de Industrialización Fronteriza.

Si la legislación actualmente propuesta es decretada, podríamos ver una repetición de los eventos que sucedieron hace cerca de 20 años. El incremento en la reubicación de trabajadores indocumentados en las ciudades fronterizas, puede muy bien seguir como secuela de la reforma de inmigración de los Estados Unidos. Esta modificación cualitativa en la población de las ciudades fronterizas, puede en cambio, otorgar preminencia a un tipo de emigración muy poco mencionada en los debates del Congreso acerca de esta cuestión: la migración de los llamados "transmigrantes". Esto es, de la gente que vive en las ciudades fronterizas y que cruza diariamente la frontera para trabajar en los Estados Unidos bajo una disposición especial por parte del Servicio de Inmigración y Naturalización.

Los transmigrantes constituyen un elemento importante de la fuerza de trabajo en las áreas norteamericanas adyacentes a la frontera con México. Ya en 1967, por ejemplo, constituían el $28 \%$ de la fuerza de trabajo total empleada en el condado de Imperial, California. Después del cierre del Programa de Braceros, el impacto de los transmigrantes en el empleo y en los salarios de las áreas fronterizas de los Estados Unidos ocupó un lugar central en los debates locales y del Congreso, acerca de la política de inmigración de los Estados Unidos. De aprobarse la actual legislación sobre migración podríamos esperar, junto con la reubicación de los antes trabajadores indocumentados en las ciudades fronterizas, un

4 Existe una gran cantidad de artículos sobre este tema. Véase por ejemplo, varios artículos presentados en The New Scholar. Vol. 9, No 1 y 2. 
incremento en el número de personas que viviendo en México tratarían de obtener empleo diario en los Estados Unidos. Actualmente, los debates han ignorado esta posibilidad; sin embargo, cuando la legislación entre en efecto como ahora ya parece ser muy probable, la transmigración que fluye directamente de las ciudades fronterizas podrá convertirse una vez más en el foco de discusión en torno a las relaciones fronterizas y la migración mexicana a los Estados Unidos. ${ }^{5}$

\section{LA FRONTERA VS. EL DESARROLLO NACIONAL}

Un bosquejo más completo en torno a lo que el futuro depara al área fronteriza, debe profundizar en la relación contradictoria que se ha desarrollado entre los objetivos propuestos por la política económica nacional que el Estado mexicano y el desarrollo regional del norte del país, considerando la cercanía que tiene con la poderosa economía de los Estados Unidos.

Los sucesos en la frontera están afectados, sustancialmente, por las relaciones económicas globales entre los Estados Unidos y México. Mientras exista una serie de problemas que son específicos del área, y que requieren algunas veces de la cooperación entre ambos países - por ejemplo, uso del agua, planeación del transporte, salud, asuntos jurídicos, cooperación policiaca, etcétera-, la solución para éstos resulta afectada por los vínculos macroeconómicos entre las dos naciones. Un buen ejemplo de ello lo constituye la drástica devaluación de la moneda mexicana desde 1980, e incluso la política actual de deslizamiento. El peso se ha desplomado de una relación de $1 / 12$ en 1976, a una de 1/350 en las últimas semanas. Este proceso tiene efectos amplificados en la economía del área fronteriza. La devaluación eleva los precios de los productos americanos que adquiere un considerable número de consumidores mexicanos que cruzan a los Estados Unidos para hacer sus compras. El efecto negativo que sufren los comerciantes de menudeo en los Estados Unidos contrasta con las ganancias de los comerciantes mexicanos al sur de la frontera. Si bien el ritmo de la inflación en México, puede algunas veces resacir el diferencial de precios de manera más o menos rápida (y existen siempre tianguis para el consumidor mexicano alerta); el resultado es un estado de incertidumbre económica. La devaluación proporciona un auge del programa de maquiladoras, ya que el salario de los trabajadores mexicanos cae junto con la moneda nacional. Sin embargo, los industriales locales, cuyas importaciones provienen de los Estados Unidos, encuentran dificultades adicionales y pueden incluso ser obligados a cerrar sus negocios debido a esos cambios de paridad monetaria.

\footnotetext{
${ }^{5}$ Véase Estudios Fronterizos. Revista del Instituto de Investigaciones Sociales, Año 1, N², UABC. México. passim.
} 
Una vez más los efectos pueden ser de diversa magnitud y significado en las diferentes partes de la frontera; pueden afectar positivamente a la industria turística de Tijuana, y de manera negativa a las empresas involucradas en la agricultura, que para su operación depende de insumos importados de los Estados Unidos.

México, después de Brasil, es la nación más endeudada del mundo. ${ }^{6}$ Gran parte de esta deuda pertenece a bancos norteamericanos. El 7 de junio de 1984, en una reunión cumbre en Londres, Estados Unidos encabezó un llamado a las naciones deudoras como México, para que abrieran sus economías a la inversión extranjera, como una medida para salir de sus crisis económicas. Varias naciones latinoamericanas (Colombia, Ecuador, Perú, Bolivia y Venezuela) han retirado recientemente una serie de restricciones legales para la entrada a la inversión extranjera directa. El que México permita una mayor intervención directa de capital estadounidense en su economía, depende de su habilidad para manejar los pagos futuros de su deuda externa.

Manteniendo esta perspectiva económica global en mente, la aprobación de la legislación restrictiva sobre inmigración en los Estados Unidos producirá, sin duda, un serio deterioro de la economía del área fronteriza. Un incremento en la reubicación de trabajadores indocumentados en el área conducirá, como sucedió al cierre del Programa Bracero, a un mayor establecimiento de empresas norteamericanas en el área. Esto ocurre ya de un modo distinto al esquema de maquiladoras generado por el PIF. La instalación de la planta Ford en Hermosillo, Sonora representa una prefiguración de lo que puede esperarse: el comienzo de una mayor integración de la región fronteriza mexicana a la economía de los Estados Unidos. En la medida en que estos sucesos se desarrollan, el gobierno central de México, al tiempo que permite un mayor relajamiento de la economía fronteriza con respecto a su celo nacionalista, podría también enfrentar la cuestión del control político sobre el área. El aumento del establecimiento de inversiones norteamericanas en la región norte del país, conlleva una mayor influencia y participación tanto de la iniciativa privada como de los intereses afines al gobierno de los Estados Unidos a nivel local, ${ }^{7}$ estatal e incluso nacional. En los últimos meses se han presentado frecuentes incidentes en las ciudades fronterizas, cuestionando el dominio del Partido Revolucionario Institucional (PRI), el partido dominante en México. La participación directa de personalidades "empresariales" en la política local de las ciudades fronterizas, fuera y dentro de la maquinaria prísta, también va en aumento.

\footnotetext{
- James D. Cockcroft. Mexico: Class Formation, Capital Accumulation and the State. New York, Monthly Review Press. 1983.

7 Jesús Tamayo. "The Northern Border of Mexico and the Crisis of 1982: A Few Preliminary Observation". En: The U.S. and Mexico: Borderland Development and the National Economies. Lay James Gibson and Alfonso Corona Rentería (eds.). Boulder: Westview Press. 1985.
} 
Recientemente se presenció un escándalo menor considerado de mal gusto por algunos funcionarios del gohierno mexicano, causado por la presencia del vicepresidente Bush en una convención nacional del PAN (Partido Acción Nacional), organización que representa los intereses de la iniciativa privada y que tradicionalmente se ha opuesto al dominio del PRI.

En conclusión, parece justo afirmar que mientras en el pasado el gobierno central de México ha tratado - no siempre con éxito- de controlar el área fronteriza económica y políticamente, el probable incremento del poder económico norteamericano en la región y el mayor peso económico de la zona fronteriza norte, podrían conducir a una inversión de papeles en un momento no muy distante en el futuro. De ahí en adelante, será la cola quien mueva al perro; la región fronteriza determinará de manera más activa las grandes tendencias sociales de México. Políticamente, existirá un amplio campo para la inestabilidad social y para una reorganización institucional del orden social; el PRI se esforzará por mantener su posición ideológica y organizativa; cualquiera que sea el grado de éxito obtenido, un desarrollo social traumático no debe ser descartado. Analíticamente hablando, los desarrollos en la frontera serán por algún tiempo la clave para comprender los desarrollos a nivel nacional; y no a la inversa. 


\section{BIBLIOGRAFIA}

COCKCROFT, James D. Mexico: Class Formation, Capital Accumulation and State. New York, Monthly Review Press, 1983.

HANSEN, Niles. The Border Economy. Austin, University of Texas Press. 1981.

HOUSE, John W. Frontier on the Rio Grande. Oxford, Clarendon Press. 1982.

PRICE, John A. Tijuana: Urbanization in a Border Culture. University of Notre Dame Press. 1973.

TAMAYO, Jesús. "The Northern Border of Mexico and the Crisis of 1982: A Few Preliminary Observations". En: The U.S. and Mexico: Borderland Development and the National Economies. Lay James Gibson and Alfonso Corona Rentería (eds.). Boulder, Westview Press. 1985. 